\title{
Target and non-target vessel related events at 10 years post percutaneous coronary intervention
}

\author{
J. J. Coughlan ${ }^{1}$ (D) Alp Aytekin ${ }^{1} \cdot$ Erion Xhepa $^{1} \cdot$ Salvatore Cassese ${ }^{1} \cdot$ Michael Joner $^{1,3} \cdot$ Tobias Koch $^{1} \cdot$ Jens Wiebe $^{1}$. \\ Tobias Lenz ${ }^{1} \cdot$ Tobias Rheude $^{1}$ - Constanza Pellegrini ${ }^{1}$ - Senta Gewalt ${ }^{1}$ - Tareq Ibrahim ${ }^{2} \cdot$ Karl-Ludwig Laugwitz $^{2,3}$. \\ Heribert Schunkert ${ }^{1,2,3} \cdot$ Adnan Kastrati $^{1,2,3} \cdot$ Sebastian Kufner ${ }^{1}$
}

Received: 4 October 2021 / Accepted: 18 January 2022 / Published online: 11 February 2022

(c) The Author(s) 2022

\begin{abstract}
Aims To define the incidence of events related to the stented vessel (target vessel related events: TVRE) and events related to non-stented vessels (non-target vessel related events: NTVRE) through to 10-year follow-up in patients post-PCI with newer generation drug eluting stents (DES).

Methods and results The current study is a post-hoc analysis of patient level data from two randomised controlled trials in Germany. Patients older than 18 years with ischemic symptoms or evidence of myocardial ischemia in the presence of $\geq 50 \%$ de novo stenosis located in the native coronary vessels were considered eligible. The endpoints of interest were TVRE (a composite of first target vessel myocardial infarction or target vessel revascularization) and NTVRE (a composite of first non-target vessel MI or non-target vessel revascularization) through to 10 years post PCI.

We included 4953 patients in this analysis. Through to 10-years post-PCI, TVRE occurred in 1238 of 4953 patients (cumulative incidence: $25.8 \%$ ) and NTVRE occurred in 1442 of 4953 patients (cumulative incidence: $30.3 \%$ ). The majority of TVRE and NTVRE were revascularization events. From 0 to 1 years, the cumulative incidence of TVRE was $15.9 \%$ and of NTVRE was $12.3 \%$. From 1 to 10 years, the cumulative incidences of TVRE and NTVRE were $11.2 \%$ and $22.4 \%$, respectively.

Conclusion At 10-year post-PCI with new generation drug eluting stents, events related to remote vessel disease progression account for a higher proportion of events than events related to the stented vessel.

Trial registration ISAR TEST 4 ClinicalTrials.gov Identifier: NCT00598676. ISAR TEST 5 ClinicalTrials.gov Identifier: NCT00598533.
\end{abstract}

Sebastian Kufner

Sebastian.Kufner@gmx.de

1 Deutsches Herzzentrum München, Technische Universität

München, Lazarettstrasse, 36, Munich, Germany

2 Klinik Und Poliklinik Innere Medizin I (Kardiologie, Angiologie Und Pneumologie), Klinikum rechts der Isar, Technische Universität München, Munich, Germany

3 DZHK (German Center for Cardiovascular Research), Partner Site Munich Heart Alliance, Munich, Germany 


\section{Graphical abstract}
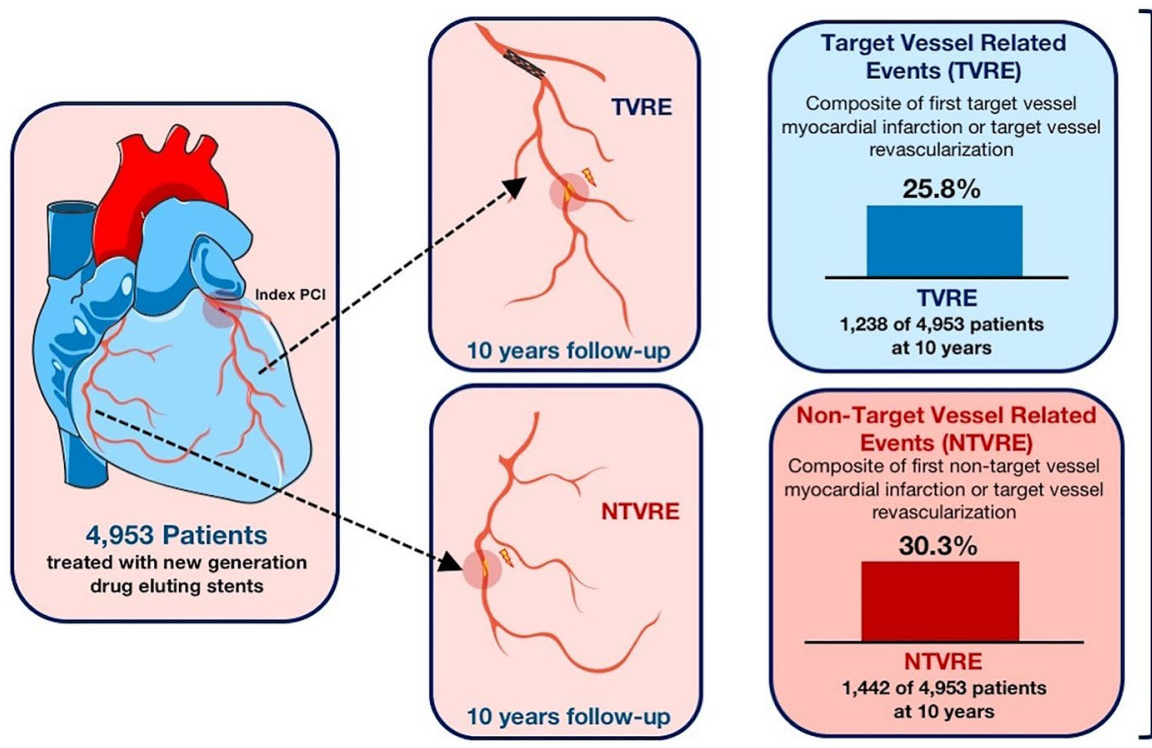
At 10-years post $\mathrm{PCl}$ with new generation drug-eluting stents, events related to remote vessel disease progression account for a higher proportion of events than events related to the stented vessel.

Keywords Percutaneous coronary intervention - Drug eluting stents · Cardiovascular outcomes · Target vessel · Atherosclerotic coronary artery disease

\author{
Abbreviations \\ PCI Percutaneous coronary intervention \\ MI Myocardial infarction \\ MACE Major adverse cardiac event \\ TVRE Target vessel related event \\ NTVRE Non-target vessel related event \\ DES Drug eluting stent \\ BP Biodegradable polymer \\ PP Permanent polymer \\ PF Polymer free \\ TVR Target vessel revascularisation
}

\section{Introduction}

Patients who undergo percutaneous coronary intervention (PCI) are at risk of subsequent cardiac events, including myocardial infarction (MI) and repeat revascularisation [1, 2]. Coronary stent trials traditionally focus on target vessel/lesion related clinical outcomes in the shorter term with a particular emphasis on outcomes in the first 12-months post-PCI [3].

It is important to consider that patients who undergo PCI are at risk of not only events related to the stented vessel (target vessel related events: TVRE) but also events related to non-stented vessels (non-target vessel related events: NTVRE) [1, 2]. While data have been published on the incidence of these event types up to 5-years post-PCI [1, 2, 4-7], their incidence at longer term follow-up has not been defined.

An analysis of TVRE and NTVRE through to 10 years after PCI in a large number of patients treated with new generation DES may be useful and help guide strategies to improve cardiovascular outcomes after PCI. Therefore, we conducted the current analysis, with the primary aim of defining the cumulative incidence of TVRE and NTVRE through to 10 years post PCI in patients enrolled in the ISAR-TEST 4 and ISAR-TEST 5 trials.

\section{Methods}

\section{Study population, study device description and enrolment criteria}

The current study is a pooled analysis of patient level data from the Intracoronary Stenting and Angiographic Results: Test Efficacy of 3 Limus-Eluting Stents (ISAR-TEST 4) and Intracoronary Stenting and Angiographic Results: Test Efficacy of Sirolimus- and Probucol-Eluting versus Zotarolimus-Eluting Stents (ISAR-TEST 5) randomised trials. The complete trial designs and 10-year clinical outcomes of both trials have been previously reported [8-11]. The ISAR-TEST 4 trial compared three different treatment arms in 2,603 patients. These were: biodegradable polymer based sirolimus 
eluting stents (BP-DES), (Yukon Choice PC, Translumina, Hechingen, Germany and Translumina Therapeutics, Dehradoon, India) $(N=1299)$, new generation permanent polymer everolimus eluting stents (PP-EES), (Xience, Abbott Vascular, Abbott Park, IL, USA), $(N=652)$ and early generation permanent polymer sirolimus eluting stents, (Cypher, Cordis Corporation, Miami Lakes, FL, USA), $(N=652)$. The ISARTEST 5 trial enrolled 3002 patients and compared clinical outcomes between new generation polymer free sirolimus and probucol-eluting stents (PF-DES) $(N=2002)$ and new generation permanent polymer-zotarolimus-eluting stents (PP-ZES) (Resolute, Medtronic Cardiovascular, Santa Rosa, California) $(N=1000)$. The polymer-free sirolimusand probucol-eluting stents consist of a pre-mounted, sandblasted, 316L stainless steel microporous thin strut ( $87-\mathrm{mm})$ stent, which is coated with a mixture of sirolimus, probucol, and shellac resin (a biocompatible resin widely used in the coating of medical tablets). This coating strategy is currently available in two devices from German manufacturers (ISAR VIVO, Translumina, Hechingen, Germany and Coroflex ISAR, B. Braun Melsungen, Berlin, Germany).

The current analysis includes all patients from the ISARTEST 4 and ISAR-TEST 5 trials, with the exception of the early generation permanent polymer sirolimus eluting stent group from ISAR-TEST 4. This group of patients were excluded from this analysis as early generation DES have been associated with a higher risk of adverse events compared to newer generation DES and their use was not felt to reflect current practice or be relevant to the study question, which is focused on new generation DES [12]. This study conforms to the declaration of Helsinki and the study protocol was approved by the ethics committee of the two participating centers in Munich, Germany (Deutsches Herzzentrum München and 1.Medizinische Klinik, Klinikum rechts der ISAR).

Enrolment criteria for both studies have been previously reported $[8,9]$. Patients older than 18 years with ischemic symptoms or evidence of myocardial ischemia in the presence of $\geq 50 \%$ de novo stenosis located in the native coronary vessels were considered eligible. Of note, patients with a target lesion in the left main stem or in cardiogenic shock were considered ineligible for both studies. Relook angiography was recommended (but not mandated) by the trial protocols at 6-8-months after the index PCI.

\section{Endpoints and definitions}

Detailed descriptions of study endpoints for both trials have been published previously $[10,11]$. The primary endpoints assessed for the current analysis were TVRE (a composite of first target vessel MI (TVMI) or target vessel revascularization (TVR)) and NTVRE (a composite of first non-target vessel MI (NTVMI) or non-target vessel revascularization (NTVR)). The target vessel was defined in this analysis as the vessel/vessels treated during the index PCI. All other vessels were classified as 'non-target vessels'. Therefore, patients could experience no events, TVRE, NTVRE or Both Events (TVRE and NTVRE) during follow-up.

Secondary endpoints included the individual components of the primary endpoints. We also analysed the median time to event for patients experiencing an endpoint through to 10 years post PCI and the interval between events for patients who experienced both a TVRE and NTVRE through to 10 years.

\section{Statistical analysis}

Continuous data are presented as means and standard deviation $( \pm \mathrm{SD})$ or medians and interquartile ranges. Categorical data are presented as counts and proportions. Differences between groups were checked for significance using an analysis of variance test (ANOVA) for continuous data. Chisquared test $(\chi 2)$ was used to check for differences between categorical variables. Survival was analyzed by the Kaplan Meier method to estimate the time to first event for each endpoint of interest. Hazard ratios were calculated using a Cox proportional hazards model after checking for fulfilment of the proportional hazards assumption as per the method of Grambsch and Therneau. The analysis of all endpoints accounted for the competing risk of death. In addition to the primary analysis, the cumulative incidences of TVRE and NTVRE were analysed as per stent polymer type, dividing patients into three groups; BP-DES, PP-DES and PF-DES. Statistical analysis was performed using the R 3.6.0 Statistical Package (The R Foundation for Statistical Computing, Vienna, Austria). A two tailed $p$ value of $<0.05$ was taken to confer statistical significance.

\section{Results}

\section{Study population}

We included 4,953 patients in this analysis. This represents $88.4 \%$ of the total cohort enrolled in the ISARTEST 4 and ISAR-TEST 5 trials. Of these patients, 1,299 (26.2\%) were treated with BP-DES, 2,002 (40.4\%) with PFDES and 1,652 (33.4\%) with PP-DES. Of these patients, 2,098 (42\%) had a clinical event (TVRE and/or NTVRE) through to 10 years follow-up. Amongst patients with an event $(N=2098), 31.3 \%(N=656)$ experienced a TVRE, $41 \%(N=860)$ a NTVRE and $27.7 \%(N=582)$ both a TVRE and NTVRE through to 10 years post PCI. Death occurred in 1,533 of 4,953 patients (31.0\%) during follow-up. 


\section{Baseline characteristics}

Baseline characteristics for the patients included in this analysis as per the event type experienced are demonstrated in Table 1. Female sex was more common in patients who experienced no events. Patients who experienced no events also tended to be older at index PCI, less commonly diabetic and had a lower frequency of triple vessel coronary artery disease. Trial protocol recommended angiography (recommended to be performed at 6-8 months after PCI) was performed less frequently in the no-events group.

\section{Angiographic and procedural characteristics}

The angiographic and procedural characteristics for the patients included in this analysis as per the event type experienced are summarized in Table 2. Patients who experienced no events had a lower proportion of complex lesions treated during the index procedure, as well as a shorter lesion and total stented length. The left anterior descending artery was more commonly treated in the group of patients who experienced no events.

\section{Primary endpoint: cumulative incidence of TVRE and NTVRE through to 10 years post $\mathrm{PCl}$}

Through to 10-years post-PCI, a TVRE occurred in 1,238 of 4,953 patients (cumulative incidence: $25.8 \%$ ) and a NTVRE occurred in 1,442 of 4,953 patients (30.3\%). These data are demonstrated in Fig. 1 and Table 3. The cumulative incidence of TVRE, NTVRE and 'Both Events' are shown in Supplemental Fig. 1.
Table 1 Baseline Characteristics as per the event type experienced during 10 -year follow-up

\begin{tabular}{lllllr}
\hline & No event & TVRE & NTVRE & Both events & $P$ value \\
\hline Patients & $(N=2855)$ & $(N=656)$ & $(N=860)$ & $(N=582)$ & \\
Age (years) & $68.0 \pm 11.3$ & $66.4 \pm 10.4$ & $66.4 \pm 10.3$ & $66.5 \pm 10.4$ & $<0.001$ \\
Female & $757(26.5)$ & $145(22.1)$ & $156(18.1)$ & $115(19.8)$ & $<0.001$ \\
Diabetes mellitus & $746(26.1)$ & $221(33.7)$ & $267(31.0)$ & $196(33.7)$ & $<0.001$ \\
Insulin-dependent & $244(8.6)$ & $84(12.8)$ & $72(8.4)$ & $74(12.7)$ & $<0.001$ \\
Hypertension & $1883(66.0)$ & $459(70.0)$ & $587(68.3)$ & $412(70.8)$ & 0.046 \\
Current smoker & $472(16.5)$ & $108(16.5)$ & $153(17.8)$ & $93(16.0)$ & 0.793 \\
Hypercholesterolemia & $1811(63.4)$ & $433(66.0)$ & $542(63.0)$ & $412(70.8)$ & 0.005 \\
Body mass index (kg/m ${ }^{2} *$ & $27.4 \pm 4.5$ & $27.8 \pm 4.7$ & $27.9 \pm 4.3$ & $27.8 \pm 4.0$ & 0.007 \\
Prior myocardial infarction & $797(27.9)$ & $192(29.3)$ & $260(30.2)$ & $199(34.2)$ & 0.021 \\
Prior aortocoronary bypass surgery & $228(8.0)$ & $67(10.2)$ & $110(12.8)$ & $77(13.2)$ & $<0.001$ \\
Number of diseased coronary vessels & & & & & $<0.001$ \\
One vessel & $568(19.9)$ & $102(15.5)$ & $60(7.0)$ & $29(5.0)$ & \\
Two vessels & $843(29.5)$ & $150(22.9)$ & $199(23.1)$ & $118(20.3)$ & \\
Three vessels & $1444(50.6)$ & $404(61.6)$ & $601(69.9)$ & $435(74.7)$ & \\
Number of lesions & $1.4 \pm 0.6$ & $1.5 \pm 0.7$ & $1.4 \pm 0.6$ & $1.6 \pm 0.8$ & $<0.001$ \\
Clinical presentation & & & & & 0.022 \\
Acute myocardial infarction & $542(19.0)$ & $111(16.9)$ & $149(17.3)$ & $102(17.5)$ & \\
Unstable angina & $613(21.5)$ & $173(26.4)$ & $194(22.6)$ & $158(27.1)$ & \\
Stable angina & $1700(59.2)$ & $372(56.7)$ & $517(60.1)$ & $322(55.3)$ & \\
Ejection fraction $(\%) *$ & $52.8 \pm 12.0$ & $53.3 \pm 10.7$ & $52.5 \pm 11.5$ & $52.5 \pm 10.8$ & 0.651 \\
Relook Angiography Performed (As & $1940(68.0)$ & $552(84.1)$ & $771(89.7)$ & $543(93.3)$ & $<0.001$ \\
$\quad$ Recommended by Trial Protocols) & & & & & \\
\hline & & & & &
\end{tabular}

Data are mean \pm standard deviation or counts $(\%)$

*Missing continuous data:

Body-mass index was not available in 6 patients ( 5 in the no event group and 1 in the both events group); ejection fraction was not available in 650 patients (356 in the no event group, 85 in the TVRE group, 116 in the NTVRE group and 93 in the both events group). The remaining continuous data were complete

TVRE target vessel related events, NTVRE non-target vessel related events 
Table 2 Angiographic and Procedural Characteristics as per the event type experienced during 10-year follow-up

\begin{tabular}{lllllr}
\hline & No event & TVRE & NTVRE & Both events & $P$ value \\
\hline Lesions & $(N=3869)$ & $(N=970)$ & $(N=1173)$ & $(N=912)$ & \\
Stent polymer type & & & & & $<0.001$ \\
Permanent polymer & $1290(33.3)$ & $309(31.9)$ & $411(35.0)$ & $319(35.0)$ & \\
Biodegradable Polymer & $964(24.9)$ & $276(28.5)$ & $295(25.1)$ & $148(16.2)$ & \\
Polymer-free & $1615(41.7)$ & $385(39.7)$ & $467(39.8)$ & $445(48.8)$ & \\
Target vessel & & & & & $<0.001$ \\
Left anterior descending artery & $1855(47.9)$ & $398(41.0)$ & $483(41.2)$ & $370(40.6)$ & \\
Left circumflex artery & $925(23.9)$ & $231(23.8)$ & $320(27.3)$ & $298(32.7)$ & \\
Right coronary artery & $1089(28.1)$ & $341(35.2)$ & $370(31.5)$ & $244(26.8)$ & \\
Chronic total occlusion & $205(5.3)$ & $49(5.1)$ & $54(4.6)$ & $67(7.4)$ & 0.037 \\
Complex morphology (B2/C) & $2744(70.9)$ & $741(76.4)$ & $878(74.9)$ & $718(78.7)$ & $<0.001$ \\
Lesion length (mm) & $15.7 \pm 9.2$ & $16.1 \pm 10.0$ & $16.2 \pm 9.4$ & $16.8 \pm 10.0$ & 0.005 \\
Vessel size (mm) & $2.8 \pm 0.5$ & $2.8 \pm 0.5$ & $2.8 \pm 0.5$ & $2.7 \pm 0.5$ & $<0.001$ \\
Total stented length (mm) & $24.9 \pm 11.6$ & $26.5 \pm 12.3$ & $25.8 \pm 12.0$ & $26.7 \pm 12.6$ & $<0.001$ \\
Percent stenosis, pre-procedure (\%) & $66.7 \pm 16.2$ & $66.9 \pm 16.2$ & $66.4 \pm 15.8$ & $67.4 \pm 15.5$ & 0.547 \\
Percent stenosis, post-procedure (\%) & $11.4 \pm 7.3$ & $12.4 \pm 8.3$ & $12.0 \pm 7.4$ & $12.4 \pm 6.9$ & $<0.001$ \\
Balloon diameter (mm) & $3.1 \pm 0.5$ & $3.0 \pm 0.5$ & $3.1 \pm 0.5$ & $3.0 \pm 0.5$ & $<0.001$ \\
Balloon: Vessel Ratio & $0.41(0.54)$ & $0.46(0.55)$ & $0.44(0.55)$ & $0.27(0.49)$ & $<0.001$ \\
Maximal Balloon Pressure (mmHg) & $15.4(3.1)$ & $15.6(3.2)$ & $15.5(3.1)$ & $15.8(3.3)$ & 0.02 \\
\hline
\end{tabular}

Data are mean \pm standard deviation or counts (\%)

$T V R E$ target vessel related events, NTVRE non-target vessel related events

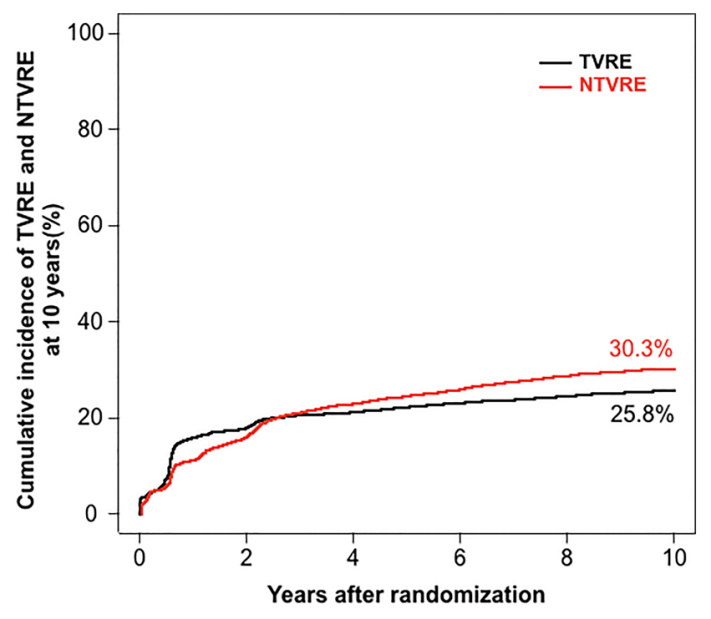

Fig. 1 Cumulative incidence of target vessel and non-target vessel related events through to 10 years post PCI

\section{Landmark analysis}

A landmark analysis was then performed to assess the cumulative incidence of TVRE and NTVRE from 0 to 1 years and $1-10$ years post PCI. From 0 to 1 years, the cumulative incidence of TVRE was $15.9 \%$ and of NTVRE was $12.3 \%$. From 1 to 10 years, the cumulative incidences of TVRE and NTVRE were $11.2 \%$ and $22.4 \%$, respectively. These data are demonstrated in Fig. 2.
Table 3 Target vessel and non-target vessel related events through to 10-years post PCI

\begin{tabular}{|c|c|c|}
\hline Total patients $(N)$ & 4953 & \\
\hline Endpoint & $\begin{array}{l}\text { Number of } \\
\text { Events }\end{array}$ & $\begin{array}{l}\text { Cumulative } \\
\text { incidence } \\
(\%)\end{array}$ \\
\hline \multicolumn{3}{|l|}{ Primary endpoint } \\
\hline Target vessel related events & 1238 & 25.8 \\
\hline Non-target vessel related events & 1442 & 30.3 \\
\hline \multicolumn{3}{|l|}{ Secondary endpoint: myocardial infarction } \\
\hline Target vessel myocardial infarction & 210 & 4.4 \\
\hline Non-target vessel myocardial infarction & 78 & 1.7 \\
\hline \multicolumn{3}{|l|}{ Secondary endpoint: revascularisation } \\
\hline Target vessel revascularisation & 1123 & 23.4 \\
\hline Non-target vessel revascularisation & 1403 & 29.5 \\
\hline
\end{tabular}

\section{Primary endpoint: As per stent polymer technology}

The frequency of both TVRE and NTVRE through 10 years was comparable for patients treated with PP-DES, BP-DES and PF-DES. This is shown in Supplemental Figs. 2, 3.

\section{Secondary endpoints: TV/NTV MI and TV/NTV revascularisation through to 10 years post $\mathrm{PCI}$}

TVMI occurred in 210 of 4,953 patients through to 10 years post PCI (4.4\%) and NTVMI occurred in 


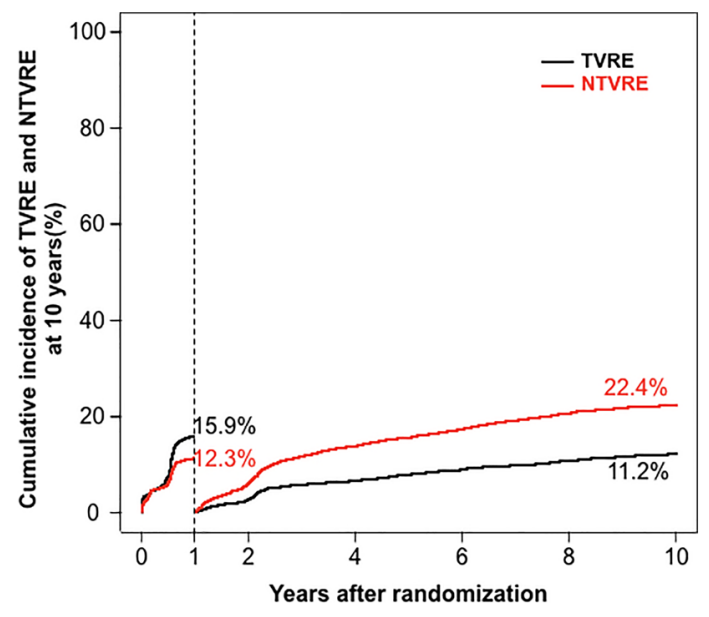

Fig. 2 Landmark analysis of target vessel and non-target vessel related events from $0-1$ and $1-10$ years post PCI

78 of 4,953 patients (1.7\%). TVR occurred in 1,123 of 4953 patients through to 10 years $(23.4 \%)$ and NTVR occurred in 1,403 of 4,953 patients $(29.5 \%)$. These data are summarised in Table 3.

\section{Cardiac death}

Cardiac Death was identified in 138 of 656 patients (21.1.\%) with isolated TVRE, 129 of 860 patients (15\%) with isolated NTVRE and 102 of 582 patients (17.5\%) with both events.

\section{Median time to first event}

The median time to first event was 205 days [inter-quartile range: 132, 468] for patients with a TVRE, 684 days [193, 1369] for patients with a NTVRE and 222 days $[148,773]$ for patients with Both Events $(p<0.001)$.

\section{Time intervals between TVRE and NTVRE}

For patients who experienced both TVRE and NTVRE during follow-up $(N=582), 205$ of 582 patients had a TVRE before a NTVRE and 208 patients had a NTVRE before a TVRE. In the remaining 169 patients, both events (TVRE and NTVRE) occurred the same day. A histogram of the time intervals between TVRE and NTVRE is demonstrated in Supplemental Fig. 4. For the majority of patients, the time interval between the two event types was less than 1 year.

\section{Discussion}

In this analysis of TVRE and NTVRE through to 10-years post-PCI with new generation DES, the main findings are as follows:

1. NTVRE account for a higher proportion of total events than TVRE at 10-year follow-up.

2. TVRE and NTVRE demonstrate distinct temporal patterns, with a higher incidence of TVRE in the first year post-PCI, followed by a higher incidence of NTVRE from year 1 to year 10 .

3. Analysis of the median time to first event echoed these results, with a shorter time to first TVRE compared to first NTVRE.

4. For the majority of patients who experience both a TVRE and a NTVRE, the time interval between these events is less than 1 year.

The current report presents the only analysis of TVRE and NTVRE through to 10-years post-PCI. It also defines for the first time the incidence of TVRE and NTVRE in patients undergoing PCI with all three newer generation DES polymer technologies (BP, PP, PF) used in current practice.

The findings of this analysis build on the sometimes divergent results of several other studies reporting on events related to stented and remote vessels/lesions post-PCI [1,2, $4,5,13]$. Cutlip et al. demonstrated that in patients receiving PCI with second generation, bare metal coronary stents, outcomes at 5 years were determined by a high rate of events related to disease progression in segments other than the stented lesion [4]. In contrast to our study, this analysis divided events into target lesion events (TLE) and non-target lesion events (NTLE). Subsequently, Stone et al. reported that in patients with ACS undergoing PCI, major adverse cardiovascular events were equally attributable to TLE and NTLE at a median follow-up of 3.4 years [1]. This study also reported that although lesions responsible for TLE were frequently angiographically mild, intravascular ultrasonography (IVUS) analysis demonstrated a combination of characteristics in these lesions, including thin-cap fibroatheroma, large plaque burden and small luminal area. In contrast to our current analysis, where all patients were treated with newer generation DES, early generation DES were implanted in only $66 \%$ of lesions in this study. Zellweger et al. subsequently reported that in an analysis of patients from 7- to 60 -months post-PCI, remote events accounted for $37.1 \%$ of all non-fatal events in a patient population treated with both bare metal stents (BMS) and early generation DES.

With regard to newer generation DES, Abdel-Wahab et al. described the incidence of non-target lesion revascularisation (NTLR) at 3-year follow-up in a large cohort of 
patients from the RESOLUTE global clinical trial program [7]. They reported that the cumulative incidence of NTLR was almost three times more frequent than TLR at 3 -years post-PCI. However, this differed to our analysis in that it focused only on TLR/NTLR and excluded patients who had both events (TLR and NTLR). In addition, the patient cohort analysed was derived from a combination of randomised controlled trial and registry data rather than exclusively from randomised controlled trial data [7].

The timing of events in our study merits further discussion. As demonstrated in the median time to first event analysis, TVRE tend to occur earlier post-PCI than NTVRE. Accordingly, TVRE accounted for a greater proportion of events than NTVRE from in the first year post-PCI. However, after the first year NTVRE began to predominate and at 10-year follow-up NTVRE account for a higher proportion of total events than TVRE. This is similar to 5-year results previously reported by Cutlip et al. [4]. It is also notable in our analysis that, after undergoing PCI to a target vessel, over one quarter of patients will have a further event related to this vessel at long term follow-up. Given the classification of events in this analysis as target vessel rather than target lesion related, these events could have been related to either the previously implanted stent or to disease progression in remote regions of the vessel that were not treated at the index PCI. Notwithstanding this limitation, it is evident from our data that both TVRE and NTVRE contribute importantly to overall event rates at long term follow-up.

Whether novel stent polymer technologies (BP, PF) influence the proportion of TVRE and NTVRE at follow-up has not been previously studied. This is important to define, because concern has been raised regarding chronic vascular inflammation with PP-DES secondary to the presence of a permanent polymer in the vessel wall [14-17]. In addition, it has been shown in animal models that focal vascular inflammation may accelerate atherosclerotic disease in remote arterial segments [18]. Therefore, it may be logical to consider that different polymer technologies could theoretically demonstrate different frequencies of non-target vessel disease progression at long term follow-up. However, in our study, the frequency of both TVRE and NTVRE at 10 years were comparable between the three studied stent polymer technologies (PP, BP, PF).

The current analysis may also serve to highlight the importance of instituting long term secondary prevention measures after PCI to improve outcomes and challenges a solely stenting/stenosis focused interventional paradigm $[19,20]$. Given that the incidence of NTVRE is unlikely to improve with iterative improvements in stent technology, novel strategies may be required, to improve long term outcomes for patients in this regard.

\section{Limitations}

The major limitation of this analysis is that it is a post-hoc analysis of pooled data from two randomised controlled trials. This is subject to the usual limitations associated with posthoc analysis and the findings should be regarded as hypothesis generating. Due to the inability to accurately ascribe mortality to TVRE or NTVRE, we were not able to include mortality or cardiac mortality in our primary endpoint. Planned angiographic follow-up at 6-8-months after PCI was recommended as part of the study protocols and this may have artificially altered the incidence of TVRE and NTVRE in comparison to standard clinical practice. In detail, 6-8-month follow-up angiography was performed in $68 \%$ of the patients in the no-events group, $84.1 \%$ of patients in the TVRE group, $89.7 \%$ of patients in the NTVRE group and $93.3 \%$ of patients in the both events group (Table 1). Intracoronary imaging or coronary physiological testing was not performed as part of the study protocols to the target or non-target vessels, and therefore, we have no information on intracoronary imaging or physiology predictors of TVRE and NTVRE. This analysis considered only the first event for both TVRE and NTVRE, and therefore, recurrent events were not accounted for. Information as to whether the procedure was staged or not was also not collected in the trial database. A final limitation of this analysis is the absence of data on rates of dual anti-platelet therapy and cardiovascular secondary prevention measures up to 10 years, both of which may have impacted on the incidence of long-term events.

\section{Conclusions}

At 10-year follow-up post-PCI, events related to non-target vessels account for a higher proportion of total events than events related to the target vessel.

Supplementary Information The online version contains supplementary material available at https://doi.org/10.1007/s00392-022-01986-4.

Author contributors JJC was primarily responsible for the drafting of this manuscript and was also involved in the conception and design of the project, analysis and interpretation of the data. AK was primarily responsible for conception and design of the project, performed statistical analysis of the data, provided revision of the manuscript for important intellectual content and is responsible for the overall content as guarantor. AA assisted with preparation of the manuscript, interpretation and analysis of the data and revision of the initial draft. SK was involved in the design and conception of the project, interpretation of the data and revised the manuscript for important intellectual content. EX, SC, MJ, TK, JW, TL, TR, CP, SG, TI, KLL and HS were involved in the conception of this project and provided their expert review and revision on the initial manuscript draft for important intellectual content. All authors reviewed and approved the final version of the manuscript prior to submission. AK is responsible for the overall content of this manuscript as guarantor. 
Funding Open Access funding enabled and organized by Projekt DEAL. These findings may be useful to help to inform strategies to improve long term cardiovascular outcomes in patients undergoing PCI. The current analysis received no funding.

\section{Declarations}

Conflict of interests MJ reports speaker fees from Biotronik, personal fees from Orbus Neich, grants and personal fees from Boston Scientific, grants and personal fees from Edwards, personal fees from AstraZeneca, personal fees from Recor, grants from AMGEN, not related to the current work, HS reports honoraria from AstraZeneca, Bayer Vital, MSD SHARP\&DOHME, Novartis, Servier, Sanofi-Aventis, Boehringer Ingelheim, Daiichi Sankyo, Amgen, Pfizer and consulting fees from AstraZeneca, Amgen, MSD SHARP\&DOHME, not related to the current work, SK reports speaker fees from AstraZeneca and speaker fees and consultant fees from Bristol Myers Squibb, not related to the current work; the other authors report no conflict of interest.

Open Access This article is licensed under a Creative Commons Attribution 4.0 International License, which permits use, sharing, adaptation, distribution and reproduction in any medium or format, as long as you give appropriate credit to the original author(s) and the source, provide a link to the Creative Commons licence, and indicate if changes were made. The images or other third party material in this article are included in the article's Creative Commons licence, unless indicated otherwise in a credit line to the material. If material is not included in the article's Creative Commons licence and your intended use is not permitted by statutory regulation or exceeds the permitted use, you will need to obtain permission directly from the copyright holder. To view a copy of this licence, visit http://creativecommons.org/licenses/by/4.0/.

\section{References}

1. Stone GW et al (2011) A prospective natural-history study of coronary atherosclerosis. N Engl J Med 364(3):226-235

2. Zellweger MJ et al (2012) Coronary artery disease progression late after successful stent implantation. J Am Coll Cardiol 59(9):793-799

3. Cutlip DE et al (2007) Clinical end points in coronary stent trials: a case for standardized definitions. Circulation 115(17):2344-2351

4. Cutlip DE et al (2004) Beyond restenosis: five-year clinical outcomes from second-generation coronary stent trials. Circulation 110(10):1226-1230

5. Chacko R et al (2009) Impact of target lesion and nontarget lesion cardiac events on 5-year clinical outcomes after sirolimus-eluting or bare-metal stenting. JACC Cardiovasc Interv 2(6):498-503

6. Spitaleri $\mathrm{G}$ et al (2018) Correlates of non-target vessel-related adverse events in patients with ST-segment elevation myocardial infarction: insights from five-year follow-up of the EXAMINATION trial. EuroIntervention 13(16):1939-1945
7. Abdel-Wahab $\mathrm{M}$ et al (2016) Incidence and predictors of unplanned non-target lesion revascularisation up to three years after drug-eluting stent implantation: insights from a pooled analysis of the RESOLUTE Global Clinical Trial Program. EuroIntervention 12(4):465-472

8. Byrne RA et al (2009) Randomized, non-inferiority trial of three limus agent-eluting stents with different polymer coatings: the Intracoronary Stenting and Angiographic Results: Test Efficacy of 3 Limus-Eluting Stents (ISAR-TEST-4) Trial. Eur Heart J 30(20):2441-2449

9. Massberg S et al (2011) Polymer-free sirolimus- and probucoleluting versus new generation zotarolimus-eluting stents in coronary artery disease: the Intracoronary Stenting and Angiographic Results: Test Efficacy of Sirolimus- and Probucol-Eluting versus Zotarolimus-eluting Stents (ISAR-TEST 5) trial. Circulation 124(5):624-632

10. Kufner S et al (2019) Ten-Year Clinical Outcomes From a Trial of Three Limus-Eluting Stents With Different Polymer Coatings in Patients With Coronary Artery Disease. Circulation 139(3):325-333

11. Kufner $\mathrm{S}$ et al (2020) 10-year outcomes from a randomized trial of polymer-free versus durable polymer drug-eluting coronary stents. J Am Coll Cardiol 76(2):146-158

12. Navarese EP et al (2014) First-generation versus second-generation drug-eluting stents in current clinical practice: updated evidence from a comprehensive meta-analysis of randomised clinical trials comprising 31379 patients. Open Heart 1(1):e000064

13. Leon MB et al (2009) Late clinical events after drug-eluting stents: the interplay between stent-related and natural historydriven events. JACC Cardiovasc Interv 2(6):504-512

14. van der Giessen WJ et al (1996) Marked inflammatory sequelae to implantation of biodegradable and nonbiodegradable polymers in porcine coronary arteries. Circulation 94(7):1690-1697

15. Finn AV et al (2007) Pathological correlates of late drug-eluting stent thrombosis: strut coverage as a marker of endothelialization. Circulation 115(18):2435-2441

16. Byrne RA, Joner M, Kastrati A (2009) Polymer coatings and delayed arterial healing following drug-eluting stent implantation. Minerva Cardioangiol 57(5):567-584

17. Joner M et al (2006) Pathology of drug-eluting stents in humans: delayed healing and late thrombotic risk. J Am Coll Cardiol 48(1):193-202

18. Lavin Plaza B et al (2020) Sustained focal vascular inflammation accelerates atherosclerosis in remote arteries. Arterioscler Thromb Vasc Biol 40(9):2159-2170

19. Park SJ, Ahn JM, Kang SJ (2011) Paradigm shift to functional angioplasty: new insights for fractional flow reserve- and intravascular ultrasound-guided percutaneous coronary intervention. Circulation 124(8):951-957

20. Mitchell JD, Brown DL (2017) Harmonizing the paradigm with the data in stable coronary artery disease: a review and viewpoint. J Am Heart Assoc 6(11):e007006 